\title{
Applications de l'électrophorèse capillaire en toxicologie clinique et médico-légale
}

\author{
Capillary electrophoresis applications \\ in clinical and forensic toxicology
}

Laurence LABAT $^{*(1)}$, Marc DEVEAUX ${ }^{(2)}$, Jean-Pierre DUBOST ${ }^{(1)}$

(1) Laboratoire de Chimie Analytique, UFR Sciences Pharmaceutiques,

3, Place de la Victoire - 33076 BORDEAUX Cedex

(2) Institut de Médecine Légale, Place Théo Varlet - 59000 LILLE

*Auteur à qui adresser la correspondance : Laurence LABAT, Laboratoire de Chimie Analytique,

UFR de Sciences Pharmaceutiques, 3, Place de la Victoire - 33076 BORDEAUX Cedex Tél : 0557571818 - Fax : 0556947090 - e-mail : laurence.labat@chimana.u-bordeaux2.fr

(Reçu le 2 mai 2000 ; accepté le 15 juin 2000)

\section{RÉSUMÉ}

Développée depuis une vingtaine d'années, l'électrophorèse capillaire (EC) est devenue une méthode de séparation efficace pour de nombreux xénobiotiques en toxicologie clinique et médico-légale. Dans cet article, les auteurs décrivent les principes fondamentaux de l'EC ainsi que les différents couplages et détecteurs spectrométriques utilisables. Les progrès récents sont détaillés et de nombreux exemples d'applications aux analyses de routine sont présentés. Les caractéristiques de cette méthode séparative (très faible volume de l'échantillon, opérations de préparations simplifiées, faible coût d'utilisation, consommation de peu de solvants polluants, robustesse de l'instrumentation) en font un puissant outil de travail en toxicologie clinique et médicolégale.

\section{MOTS-CLÉS}

Electrophorèse capillaire, Toxicologie clinique, Toxicologie médico-légale, Revue.

\section{SUMMARY}

Capillary electrophoresis (CE) which appeared in the early 1980 's, is now emerging as an effective method for separation of various substances in clinical and forensic toxicology. This paper describes the basic principles of $C E$, including on-line hyphenation and spectral identification. Recent advances and breakthrough to routine assays for drugs in biological and post-mortem specimens are presented. It is concluded that the specificity of this particular separation mechanism as well as its distinct features (small sample size, minimal sample preparation, negligible running costs, ruggedness of instrumentation, pollution-controlled) makes $C E$ a powerful toy of investigation in clinical toxicology and forensic sciences.

\section{KEY-WORDS}

Capillary electrophoresis, Clinical toxicology, Forensic toxicology, Review. 


\section{Abréviations}

Les abréviations des termes anglo-saxons les plus couramment utilisés en électrophorèse capillaire sont indiquées en italique.

CEC Chromatographie électrocinétique ou électrochromatographie

CECM Chromatographie électrocinétique micellaire (micellar electrokinetic capillary, MEKC)

CG-SM Chromatographie en phase gazeuse couplée à la spectrométrie de masse

CLHP Chromatographie liquide haute performance

DBD Détecteur à barette de diodes

EC Electrophorèse capillaire (capillary electrophoresis, CE)

ECZ Electrophorèse capillaire de zone (capillary zone electrophoresis, CZE)

EZHP Electrophorèse de zone haute performance (high performance capillary electrophoresis, $H P C E$ )

FEO Flux électroosmotique (electroosmotic flow, EOF)

FIL Fluorescence induite par laser (laser induced fluorescence, LIF)

IES Ionisation par electrospray (electrospray ionisation, ESI)

SM Spectrométrie de masse.

\section{1- Introduction}

Les premières tentatives de séparation par électrophorèse capillaire $(\mathrm{EC})$ ont été réalisées sur des tubes de 3 mm de diamètre par Hjerten en 1967 (1). Par la suite, la diminution de la taille des tubes a contribué à la réduction du gradient de température et à la dissipation de la chaleur produite par effet Joule. Cependant, un certain nombre de difficultés technologiques concernant la séparation (le milieu devenant plus résistant) et la détection (une plus grande sensibilité devenant indispensable) ont du être résolues. En 1981, Jorgerson franchit une nouvelle étape, introduisant l'utilisation de tubes capillaires en silice fondue de $75 \mu \mathrm{m}$ de diamètre interne, avec détection par fluorescence directement à travers le tube (2). L'électrophorèse de zone à haute performance (EZHP) devenait alors une méthode utili- sable en chimie analytique. Depuis, le développement technologique de l'EZHP s'est poursuivi dans le sens de la miniaturisation, parallèlement à la microchromatographie en phase liquide apparue dans les années 1980. Des techniques hybrides sont alors apparues, comme la chromatographie électrocinétique micellaire $(\mathrm{CECM})$ et la chromatographie électrocinétique ou électrochromatographie (CEC) et il est souvent difficile de définir une frontière nette entre ces deux techniques.

L'équipe de Weinberger et Lurie a été la pionnière de l'utilisation de l'EC en toxicologie analytique en montrant qu'il était possible de séparer 18 substances différentes (3). Thormann et ses collaborateurs ont ouvert la voie du dosage de médicaments et de drogues illicites dans des échantillons biologiques par EC (4-7).

Depuis une dizaine d'années les domaines d'applications sont de plus en plus variés, s'orientant non seulement vers la toxicologie clinique mais également vers la toxicologie médico-légale (8-9). L'équipe de Thormann est certainement encore la plus active dans l'analyse des milieux biologiques (10-12) et il décrit dans une revue de la littérature sur les années 1997 et 1998 les progrès récents de l'EC et les applications en routine en toxicologie clinique et médico-légale. Des études plus spécifiques se développent : l'EC est en train de devenir l'approche la plus commune pour l'analyse chirale de nombreux médicaments et substances illicites (13-25).

La séparation en EC repose essentiellement sur la mobilité électrophorétique, le coefficient de partage entre les phases, les constantes d'ionisations et la taille des molécules. Cette diversité de paramètres est une des raisons de la richesse d'applications de cette technique. Comparée aux techniques chromatographiques, $l^{\prime} E C$ devient une technologie très attrayante par son efficacité, sa sélectivité, sa facilité d'emploi, sa souplesse et sa rapidité d'exécution. D'autre part, l'utilisation de très faibles volumes d'échantillon, la faible consommation de réactifs, le moindre coût des consommables et en particulier des capillaires, peuvent faire préférer cette technique aux techniques chromatographiques.

Dans cet article, nous rappellerons les principes et les notions fondamentales de l'EC, puis nous ferons le point sur la place actuelle de cette technique dans l'analyse en toxicologie clinique et en toxicologie médicolégale. 


\section{2- Principe et notions fon- damentales de l'électro- phorèse capillaire}

\section{1 - Principe}

La figure 1 représente le schéma de principe de l'appareillage nécessaire à la mise en œuvre d'une analyse en EC. Le capillaire est rempli avec un électrolyte. L'échantillon contenant les molécules à séparer est injecté à l'une des deux extrémités. Ces dernières sont ensuite immergées dans deux récipients contenant ce même électrolyte et recevant les électrodes. Sous l'action d'un champ électrique, les constituants se séparent progressivement au sein de l'électrolyte par différence de vitesses de migration et sont détectés à proximité de l'autre extrémité du capillaire (26). Des voltages très importants (plusieurs dizaines de $\mathrm{kV}$ ) sont utilisés pour séparer les molécules sur la base de leur différence de rapport charge/taille. Le tracé obtenu en EC est appelé électrophorégramme.

Dans l'électrophorèse capillaire de zone (ECZ), la séparation résulte de la migration électrophorétique (mouvement des molécules chargées vers l'électrode de polarité inverse) et du flux électroosmotique (FEO) (mouvement d'électrolytes lié à la charge interne du capillaire et du potentiel appliqué).

La vitesse de migration électrophorétique $v_{e}$ s'exprime en fonction de la mobilité électrophorétique de l'ion $\mu_{e}$ et du champ électrique $E$ :

$v_{e}=\mu_{e} . E$

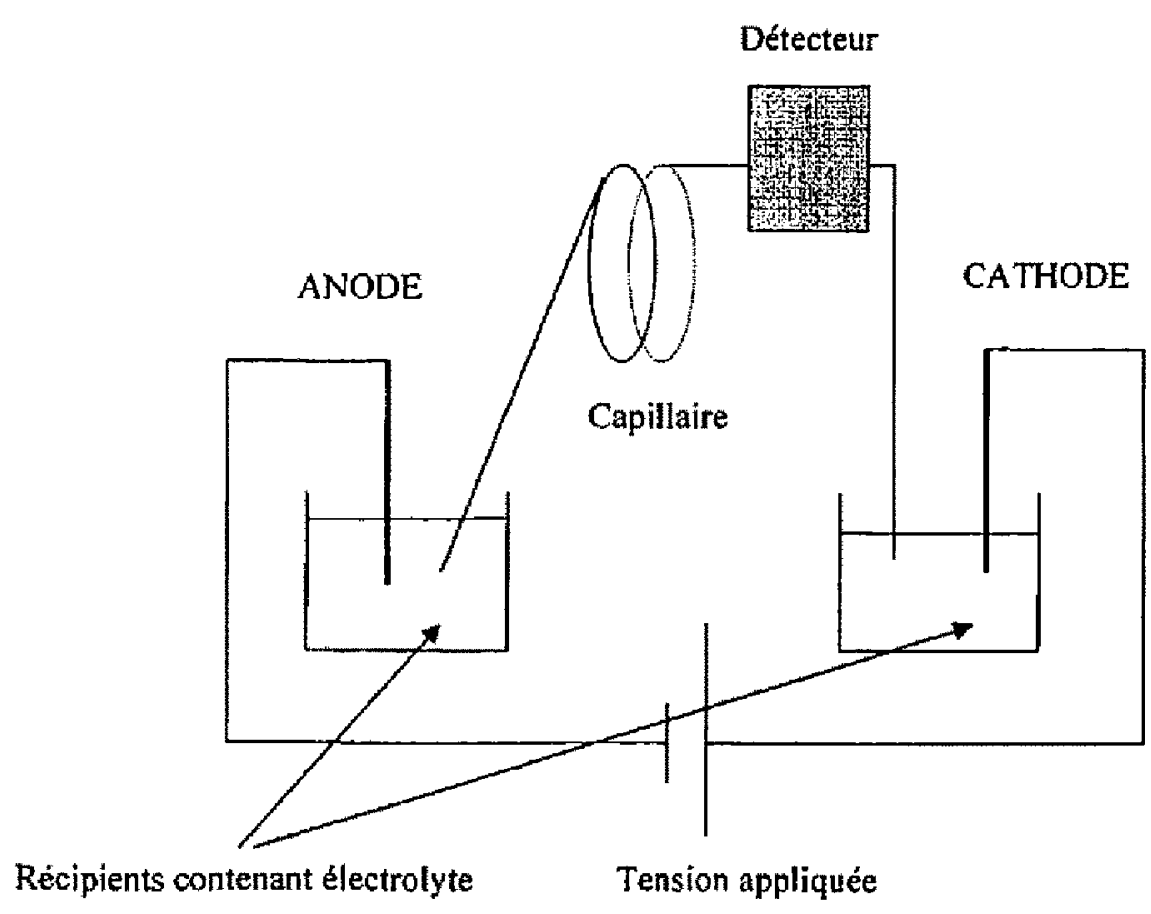

Figure 1 : Schéma de l'appareillage de l'électrophorèse capillaire.
Le champ électrique est fonction de la tension appliquée et des caractéristiques du capillaire. La mobilité électrophorétique est constante pour une espèce ionique donnée. Elle s'exprime en fonction de la charge ionique $q$, de la viscosité de la solution $\eta$ et du rayon ionique $r$ :

$\mu_{e}=q / 6 \pi \eta r$

Pour une valeur de $\mathrm{pH}$ supérieure à 2 , le FEO est généré par une couche d'ions négatifs sur la surface du capillaire provenant de la dissociation des sites silanols chargés négativement ( $\mathrm{SiO}$ ) qui attirent les ions positifs de l'électrolyte. La présence de cette double couche d'ions crée une différence de potentiel appelée potentiel zêta $\zeta$. L'application d'une tension entre les deux extrémités du capillaire est à l'origine de la migration de ces ions positifs vers la cathode et d'un flux d'eau dans la même direction. La vitesse $v_{e o}$ de ce FEO peut être exprimée en fonction de la constante diélectrique $\varepsilon$ du potentiel $\zeta$, de la viscosité de la solution $\eta$ et du champ électrique $E$ par l'équation :

$\nu_{e o}=\varepsilon \zeta E / 4 \pi \eta$

Le FEO est dans la plupart des cas dirigé de l'anode vers la cathode et il peut être déterminé de façon empirique en injectant un marqueur neutre (en général de l'acétone) dont on mesure le temps de migration.

Dans le cas le plus classique d'une injection à l'anode et d'une détection à la cathode, il est possible d'analyser des anions en EC à condition que leur mobilité résultant du FEO soit plus élevée que leur mobilité électrophorétique. La mobilité électrophorétique des cations est augmentée par le FEO et les anions sont paradoxalement entraînés aussi vers la cathode par le FEO. Ainsi, le FEO entraîne vers la cathode d'abord les molécules chargées positivement, puis les molécules neutres, et enfin les molécules chargées négativement (27). De plus, les cations qui possèdent le rapport charge/masse le plus élevé migrent avant les cations dont le rapport est plus faible. Ce rapport peut être modifié en ajustant le $\mathrm{pH}$ du tampon de migration, affectant ainsi l'état d'ionisation et donc la mobilité électrophorétique. Le FEO peut aussi être modifié, selon la puissance du champ électrique, le tampon (composition, $\mathrm{pH}$, force ionique, viscosité) et les caractéristiques de la surface du capillaire.

\section{2 - Préparation des échantillons}

Une des particularités de l'EC est l'injection de très faibles volumes. De l'ordre de quelques nanolitres, ils permettent de travailler théoriquement à partir de petits volumes de prélèvements mais une étape de pré- 
concentration de l'échantillon est souvent nécessaire et des volumes initiaux de $0,5 \mathrm{~mL}$ à $2 \mathrm{~mL}$ sont couramment utilisés. En théorie, un volume de $10 \mu \mathrm{L}$ serait suffisant bien qu'on en injecte 1000 fois moins. Or, il n'existe pas actuellement de flacons porte-échantillons de contenance inférieure à $50 \mu \mathrm{L}$, et il est nécessaire de remplir complètement ces flacons pour assurer la reproductibilité de l'injection.

La préparation des échantillons est importante si l'on considère que certains constituants des échantillons peuvent interagir avec la paroi des capillaires, modifiant les caractéristiques du FEO et par conséquence l'efficacité et la reproductibilité.

Tenant compte des paramètres précédemment abordés, différentes approches ont été décrites pour la préparation des échantillons en vue de l'analyse des médicaments et drogues illicites en EC.

\subsection{1 - Injection directe sans pré-traitement}

Malgré la présence de composés endogènes et exogènes pouvant créer des interférences et malgré les fortes concentrations en sels et en protéines dans les échantillons biologiques, on peut se contenter d'une injection directe après une simple filtration/centrifugation pour éliminer les particules susceptibles d'obstruer le capillaire. Thormann décrit l'analyse des barbituriques en $\mathrm{EC}$ avec l'injection directe du sérum (4). L'analyse en EC (EC-UV et EC-SM) d'analgésiques et d'anti-inflammatoires (paracétamol, acide acétylsalicylique, antipyrine, ibuprofène, naproxène, kétoprofène et propyphénazone) après injection directe d'urine est tout à fait possible en raison d'une séparation suffisante (28). Cependant, l'injection directe de l'échantillon reste peu appliquée à l'analyse toxicologique de prélèvements biologiques en raison de la faible concentration des xénobiotiques. Ce mode d'injection reste cependant plus adapté à la CECM en raison de l'effet dénaturant du dodécyl sulfate de sodium (très couramment utilisé en CECM) sur les protéines du sérum.

\subsection{2 - Dilution de l'échantillon}

Pour minimiser les interférences de la matrice, la technique la plus simple est la dilution de l'échantillon avec un tampon de force ionique 10 à 20 fois inférieure à celle du tampon de migration ou avec de l'eau pure. L'opposition entre les très faibles concentrations en protéines et en ions de l'échantillon et la molarité élevée du tampon est à l'origine d'un effet d'amplification du champ électrique ou stacking effect. Mais cette dilu- tion s'oppose bien sûr à la sensibilité de la méthode et n'a été que rarement utilisée pour l'analyse des dérivés amphétaminiques, morphiniques et de la caféine dans l'urine et le sérum (29) ou dans le cas très particulier du dosage du potassium dans le vitré de l'œeil (30). Cette technique reste la seule utilisée pour l'analyse des substances saisies (ecstasy, amphétamines, héroïne, cocaïne) avec des dilutions par des solutions basiques ou acides (31-34). En effet, la concentration des différents composants y est importante, sans commune mesure avec celle que l'on retrouve dans les échantillons biologiques.

Ces approches (injection directe et dilution de l'échantillon) sont rapides, simples, peu coûteuses et utilisables en toxicologie clinique. En toxicologie médicolégale, des pré-traitements de l'échantillon plus sélectifs sont généralement préférés de façon à diminuer le bruit de fond dû à la matrice.

\subsection{3 - Précipitation par un solvant organique}

La précipitation des protéines par un solvant organique suivie d'une centrifugation et de l'injection du surnageant est un des plus simples et des plus classiques mode de préparation d'échantillons en chromatographie. Elle est particulièrement bien adaptée à l'EC. L'avantage de cette préparation est important pour des composés peu solubles en tampon de migration aqueux. Cependant, l'addition de solvants organiques (alcools, acétonitrile) augmente le pouvoir d'évaporation de l'ensemble de l'échantillon, présente l'inconvénient majeur de diluer l'échantillon et a un effet dénaturant qui diminue la conductivité du mélange (35). Cette technique ne peut être utilisée en mode micellaire et son utilisation reste limitée à l'ECZ. Ce mode de préparation n'a pas encore été utilisé pour l'analyse toxicologique d'échantillons biologiques ou de substances.

\subsection{4 - Extractions en phase liquide et en phase solide}

Les méthodes d'extraction en phase liquide et en phase solide sont utilisées pour préparer les échantillons à analyser en EC, comme en CLHP ou en CG. Ces techniques de concentration s'ajoutent à celles pouvant être obtenues par l'injection électrocinétique ou par le processus d'amplification de champ (voir paragraphe 2.3.3).

Des exemples sont donnés dans le tableau I. 


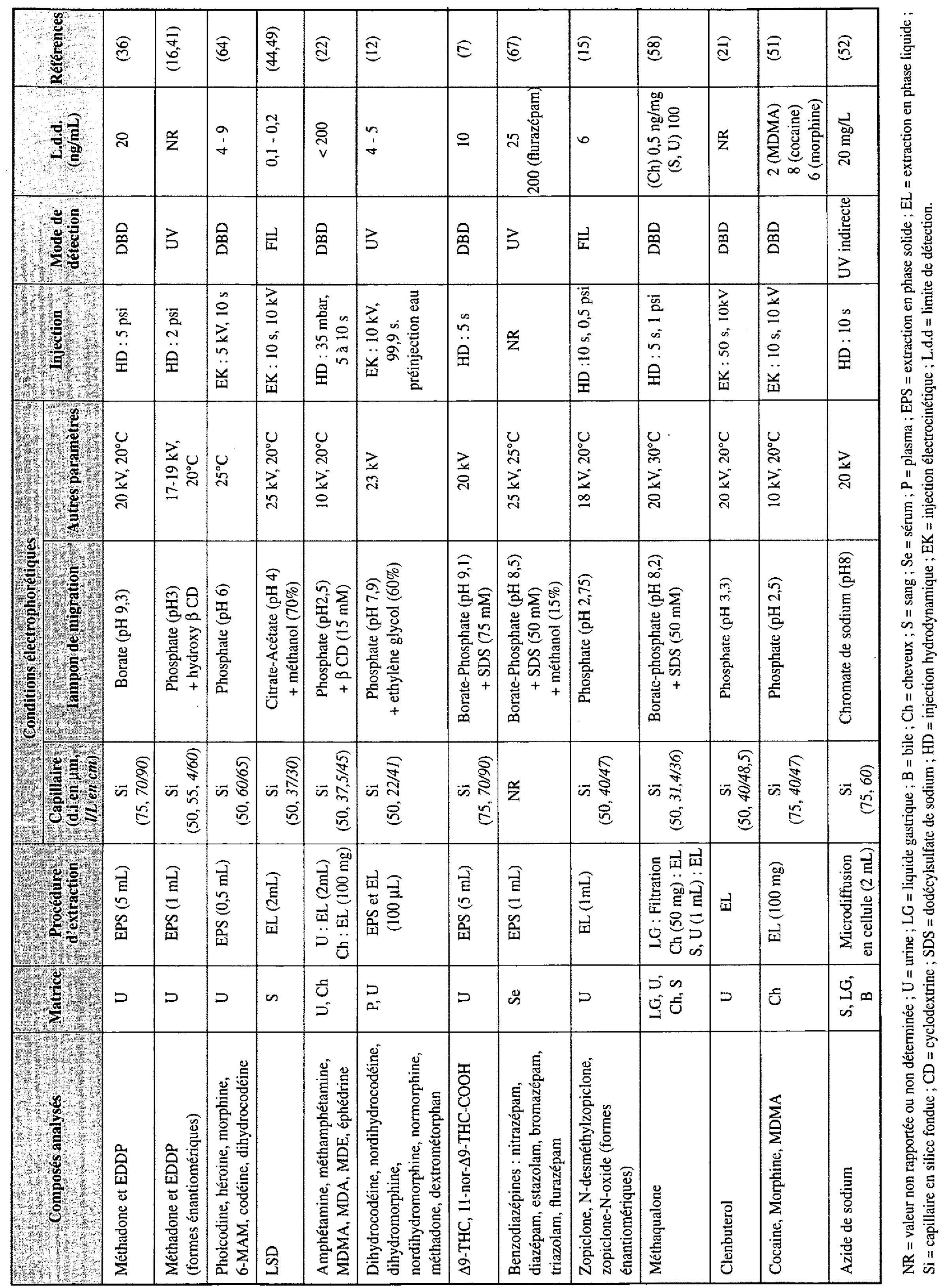




\section{3 - Conditions opératoires}

\subsection{I - Nature et conditionnement du capillaire}

L'élément essentiel de l'EC est le capillaire en silice fondue dans lequel se font la séparation et la détection. Il doit donc être chimiquement et physiquement inerte, ne doit pas absorber dans l'ultraviolet dans la fenêtre de détection (en mode de détection UV ou DBD) et ne doit pas adsorber de solutés à l'origine d'une augmentation de température par effet Joule. Il doit être inséré dans un support, la cartouche qui est elle même introduite manuellement dans l'appareil.

Le choix du capillaire dépend principalement de l'application et à un moindre degré, de l'instrumentation utilisée. Des capillaires en silice fondue non traitée sont classiquement utilisés en toxicologie analytique. Ils sont de longueur et de diamètre variables. La longueur d'un capillaire se définit par deux paramètres $L$ et $l$, qui sont respectivement la longueur totale et la longueur efficace (longueur entre l'électrode d'injection et la cellule de détection). Le plus fréquemment, 1 varie de 40 à $60 \mathrm{~cm}$ (tableaux II et III), mais Molteni (36) utilise un capillaire de $90 \mathrm{~cm}$ pour le dosage de la méthadone dans les urines et Ramseis (37) de $100 \mathrm{~cm}$ pour la séparation de dérivés amphétaminiques et analogues dans les urines. Des capillaires plus courts $(23 \mathrm{~cm})$ sont quelquefois utilisés pour des screening rapides (29). Deux diamètres internes de capillaires sont classiquement décrits dans la littérature pour des applications toxicologiques : 50 et $75 \mu \mathrm{m}$. Tagliaro (8) a montré que la sensibilité était meilleure avec un diamètre de $75 \mu \mathrm{m}$, tout en diminuant le potentiel appliqué entre les deux électrodes afin de conserver des intensités de courants pas trop élevées $(<100 \mathrm{~mA})$.

Les capillaires sont chargés négativement, attirant les cations en solution, y compris les protéines. De nombreuses stratégies minimisant ou empêchant les interactions paroi-solutés ont été développées : utilisation de tampon à $\mathrm{pH}$ élevé ou au contraire faible pour lesquels les groupements silanols ne sont pas dissociés, utilisation de tampons à force ionique élevée, d'additifs dans les tampons (agents non ionisés masquant les charges présentes à la surface du capillaire diminuant ou annulant le FEO). L'adsorption de surfactifs chargés (bromure de cétyl triméthyl ammonium) peut également modifier la surface du capillaire, inverser sa charge et ainsi inverser le sens du FEO. Modifier la surface du capillaire est une approche simple mais la modification n'est pas toujours stable en dehors d'une certaine fourchette de $\mathrm{pH}$ et peut altérer la reproductibilité des résultats.
A coté des capillaires en silice fondue, on notera l'utilisation très ponctuelle de capillaires recouverts de polyacrylamide permettant une séparation rapide de différents barbituriques et benzodiazépines (38) ou de capillaires recouverts de charges neutres (eCAPTM, Beckmann ou DB-17, J\&W Scientific) pour la séparation des formes énantiomèriques d'amphétamines (22).

Les caractéristiques de la surface interne du capillaire en silice fondue, c'est à dire l'état d'ionisation des groupements silanols, est primordial dans le développement analytique. La façon la plus simple d'obtenir des résultats reproductibles est de procéder à un entretien régulier du capillaire entre chaque injection ainsi qu'en début et fin d'analyse (tableau II). En général, ce traitement comprend une première étape de rinçage par une base forte $(\mathrm{NaOH}$ à $0,1 \mathrm{M}$ ou $1 \mathrm{M})$ afin de régénérer les sites silanols. Cette étape doit être suivie par un lavage à l'eau distillée puis par un conditionnement avec du tampon de migration $(23,34,39)$. C'est à ce moment qu'un traitement du capillaire peut être réalisé par des agents de recouvrement comme le polyacrylamide ou les polyéthylèneglycols. Il est également conseillé d'effectuer un rinçage rapide par de la soude puis du tampon entre deux injections $(15,36$, 40). Après chaque série de 5 à 10 injections, le tampon de migration est changé $(36,41)$. Une électrolyse du tampon se produit au cours des séparations sous l'effet de la tension appliquée : elle se traduit par une acidification de l'électrolyte du côté anodique et une alcalinisation du côté cathodique. Ce changement dans la valeur de $\mathrm{pH}$ varie en fonction du pouvoir tampon, de la force ionique de l'électrolyte et de la durée de la séparation (42). On terminera une série d'analyses en injectant de l'air dans le capillaire après rinçage de celui-ci par de l'eau distillée. Les détails sont donnés dans le tableau II.

Tableau II : Étapes de conditionnement du capillaire en silice fondue.

\begin{tabular}{|c|c|c|c|}
\hline $\begin{array}{l}\text { Duré des étapes de } \\
\text { rinçage du capillaire }\end{array}$ & $\begin{array}{l}\text { Debut d'analyse } \\
\qquad(23,34,39)\end{array}$ & $\begin{array}{l}\text { Fin d'analyse } \\
(56)\end{array}$ & $\begin{array}{l}\text { Entre chaque injection } \\
\qquad(15,36,40)\end{array}$ \\
\hline Eau distillée & $5 \mathrm{~min}$. & & \\
\hline $\mathrm{NaOH}(1 \mathrm{M})$ & 3 min. à 10 min. & & \\
\hline $\mathrm{NaOH}(0,1 \mathrm{M})$ & $5 \mathrm{~min}$. à $10 \mathrm{~min}$. & & $40 \mathrm{sec}$. à $1 \mathrm{~min}$. \\
\hline Eau distillée & $5 \mathrm{~min}$. à $10 \mathrm{~min}$. & $5 \mathrm{~min}$ aे $10 \mathrm{~min}$. & \\
\hline Tampon de migration & $10 \mathrm{~min}$. à $20 \mathrm{~min}$. & & $100 \mathrm{sec}$. à $2 \mathrm{~min}$. \\
\hline Air & & $5 \mathrm{~min}$ & \\
\hline
\end{tabular}




\begin{tabular}{|c|c|c|c|c|c|c|c|}
\hline$\frac{\tilde{g}}{\tilde{w}}$ & 屃 & $\stackrel{\widetilde{E}}{\widetilde{E}}$ & స్ & 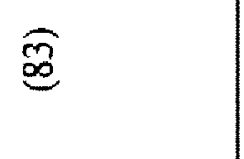 & 后 & 鸽 & 婑 \\
\hline $\begin{array}{l}8 \\
\frac{5}{6} \\
\frac{5}{2}\end{array}$ & 兽 & 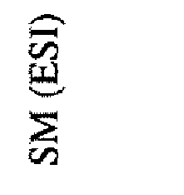 & 3 & 合 & 3 & 3 & 鳋 \\
\hline$\frac{\frac{g}{g}}{s}$ & 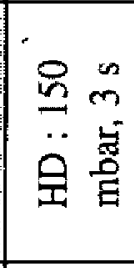 & 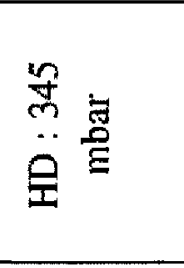 & 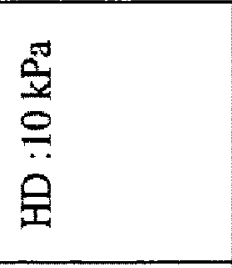 & 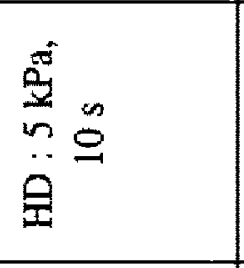 & \begin{tabular}{|l|}
$n$ \\
$n$ \\
$z$ \\
$n$ \\
$\ddot{n}$ \\
$\ddot{y}$ \\
\end{tabular} & 总 & 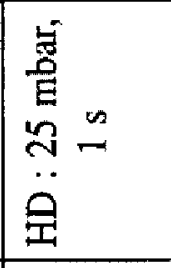 \\
\hline $\begin{array}{l}\frac{g}{3} \\
\frac{2}{2} \\
\frac{2}{2} \\
\frac{3}{3}\end{array}$ & $\begin{array}{l}0 \\
0 \\
0 \\
0 \\
3 \\
0 \\
0\end{array}$ & $\begin{array}{l}0 \\
0 \\
i \\
3 \\
3 \\
n \\
n\end{array}$ & $\begin{array}{l}0 \\
0 \\
0 \\
\frac{0}{1} \\
\frac{1}{1}\end{array}$ & $\begin{array}{l}0 \\
0 \\
0 \\
0 \\
0 \\
0 \\
0 \\
1 \\
1\end{array}$ & 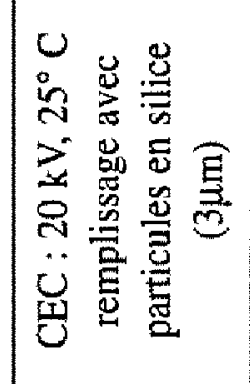 & $\begin{array}{l}0 \\
0 \\
0 \\
\hat{\infty} \\
\vdots \\
\vdots \\
\vdots\end{array}$ & $\begin{array}{l}0 \\
0 \\
i n \\
i \\
z \\
⿱ 亠 䒑 \\
0\end{array}$ \\
\hline$\frac{2}{2}$ & 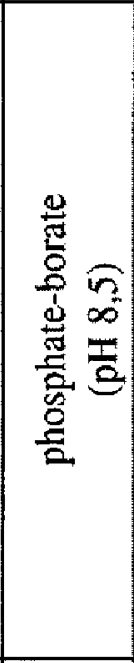 & 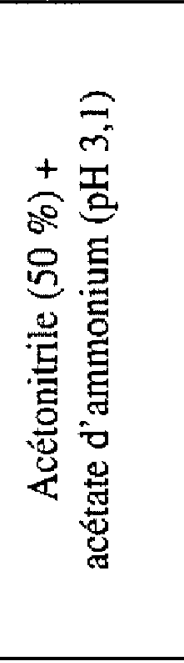 & 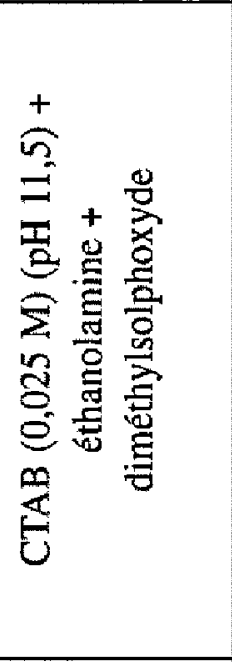 & 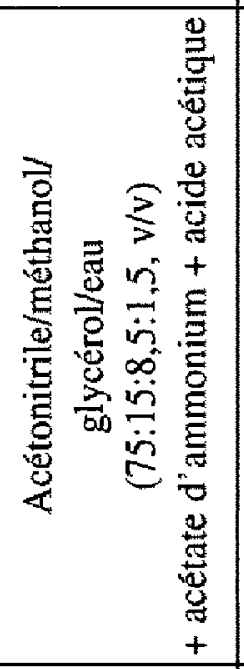 & 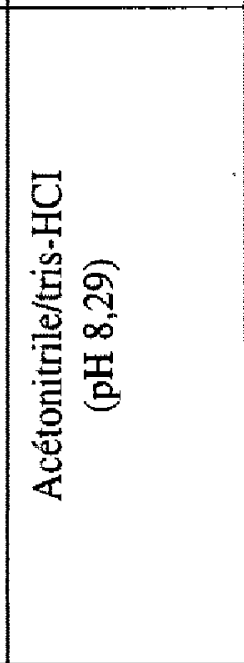 & 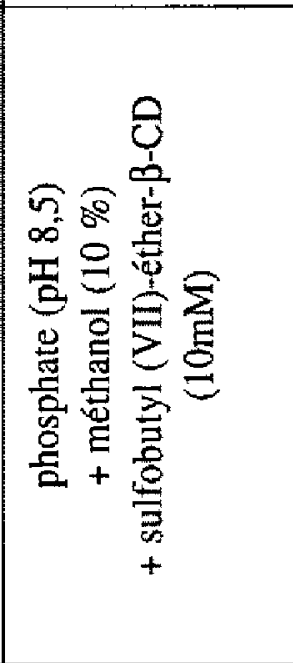 & 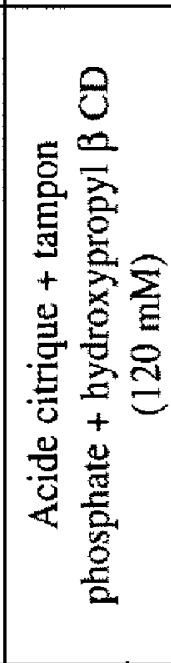 \\
\hline Ge & 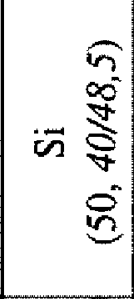 & 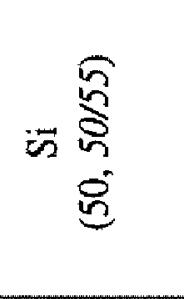 & 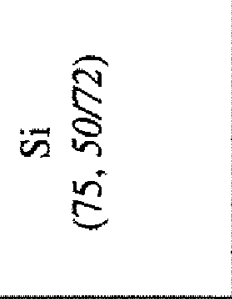 & 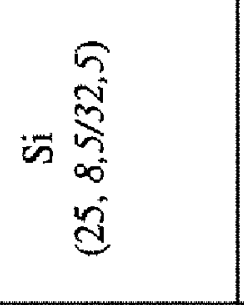 & 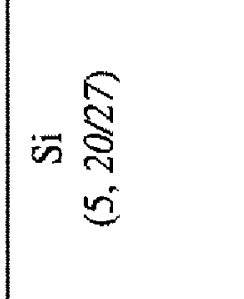 & 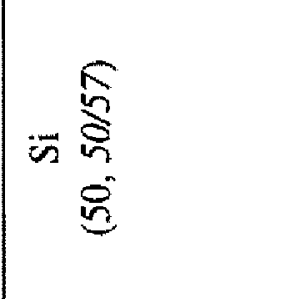 & 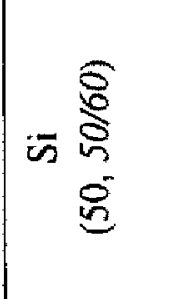 \\
\hline 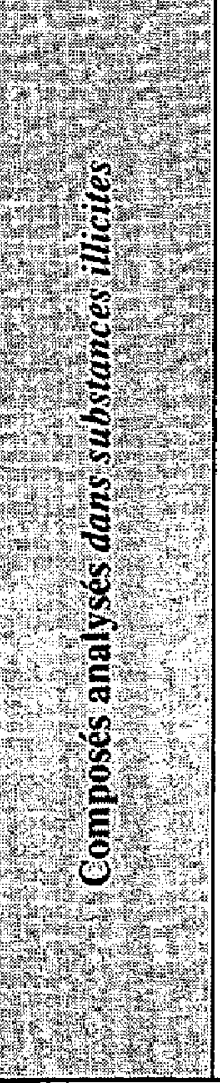 & $\begin{array}{l}0 \\
0 \\
0 \\
0 \\
0 \\
0\end{array}$ & 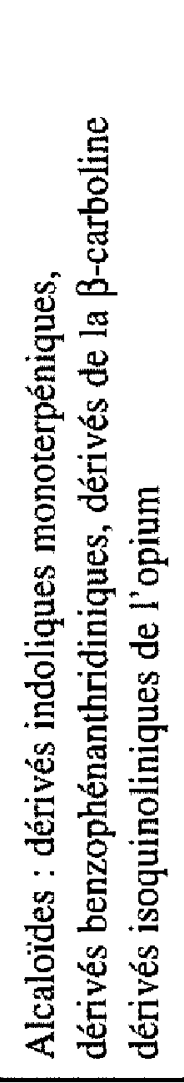 & 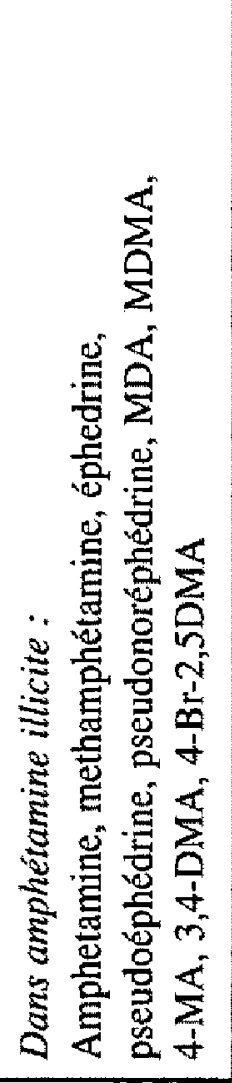 & 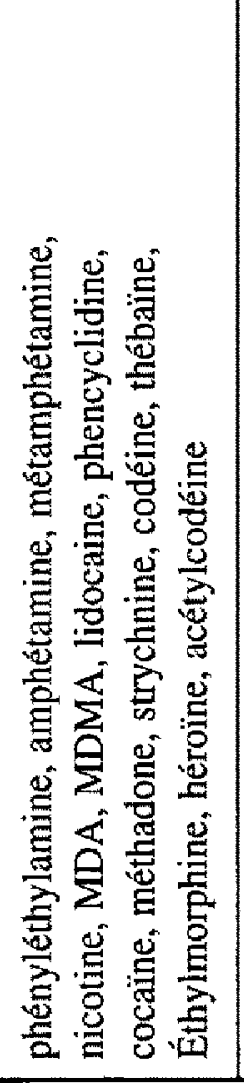 & 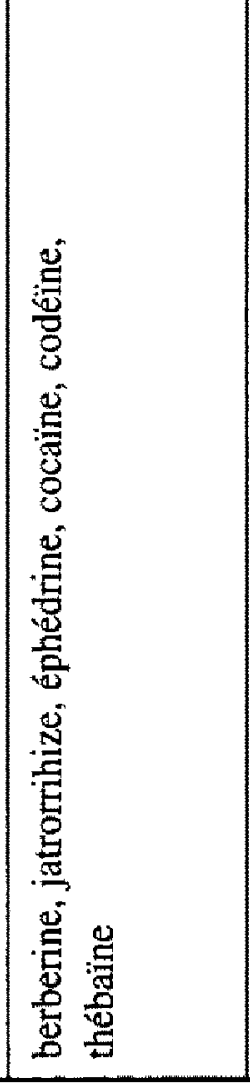 & 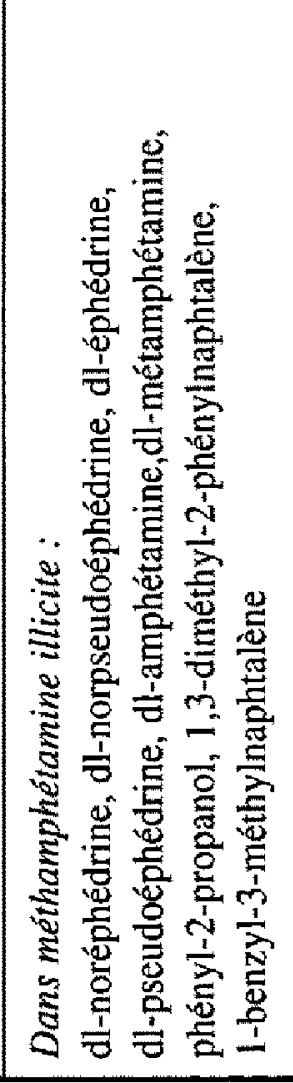 & 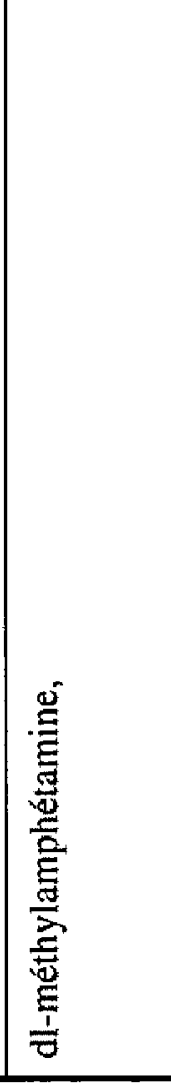 \\
\hline
\end{tabular}




\subsection{2 - Modes d'électrophorèse capillaire utilisés en toxicologie}

Les différentes techniques en EC utilisées pour l'analyse en toxicologie clinique et médico-légale sont l'électrophorèse capillaire de zone (ECZ), l'électrophorèse capillaire de type micellaire (CECM) et l'électrochromatographie capillaire (CEC).

\subsubsection{1 - Électrophorèse capillaire de zone}

Les caractéristiques fondamentales de l'ECZ sont l'homogénéité de la solution tampon et la constance du champ électrique sur toute la longueur du capillaire. La séparation est basée sur des différences de mobilités électrophorétiques dues à des différences de taille et de charge à un $\mathrm{pH}$ donné. Seules les molécules neutres ne peuvent pas être séparées. Les analyses en $\mathrm{ECZ}$ peuvent être réalisées en l'absence ou en présence de phénomène d'électro-osmose sachant que la présence du FEO permet une analyse simultanée des anions et des cations (voir paragraphe 2.1).

L'ECZ est la technique la plus couramment utilisée en toxicologie $(12,21,23,32-34,36,43-52)$. Des exemples sont donnés dans les tableaux I et III.

\subsubsection{2 - Électrophorèse capillaire électrocinétique micellaire ou chromatographie électrocinétique micellaire}

La CECM est une combinaison des techniques électrophorétiques et chromatographiques. On distingue : une phase aqueuse et une phase pseudo stationnaire, la phase micellaire. Dans sa forme la plus commune, cette dernière phase est constituée par des tampons contenant des surfactants chargés (en général le sulfate de dodécyl sodium ou le taurodeoxycholate de sodium) (3, 53-55). Les surfactants sont ajoutés à une concentration supérieure à leur concentration micellaire critique. La phase micellaire et le tampon aqueux ayant des vitesses de migration différentes, les solutés non ionisés peuvent être séparés en fonction de leur coefficient de partage entre les deux phases. Développée à l'origine pour la séparation de composés neutres sans mobilité électrophorétique $(54,56)$, la CECM permet de séparer des composés chargés de mobilités électrophorétiques voisines (57). La séparation de ces solutés ionisés est également fonction de la charge et donc de la composition du tampon, du $\mathrm{pH}$ et de la présence de modificateurs organiques. L'effet dénaturant de détergents sur les protéines des matrices permet ainsi d'injecter directement de l'urine et du plasma $(4,58)$.

L'ECZ et la CECM avec ajout de sélecteurs chiraux comme les cyclodextrines, sont des méthodes simples et peu coûteuses utilisées pour la séparation de dérivés énantiomèriques $(13,16,18,22,59,60)$ ou de mélanges de molécules chimiquement très proches comme les antidépresseurs tricycliques (57).

\subsubsection{3 - Électrochomatographie capillaire}

Dans la CEC, le capillaire est rempli avec un support chromatographique, ce qui fait de cette technologie une méthode hybride entre la CLHP et l'EC. La phase liquide est en contact avec la silice du capillaire et avec les surfaces des particules de la phase solide chromatographique. Le FEO se produit dans les doubles couches de charges qui existent au niveau des interfaces solideliquide du capillaire et des particules.

\subsection{3 - Modes d'injection}

En raison de la taille très réduite du capillaire, les volumes d'échantillons injectés (de l'ordre de quelques nanolitres) doivent être parfaitement contrôlés. Les automates actuellement commercialisés assurent une reproductibilité à l'injection supérieure à $2 \%$. En général, deux modes d'injection peuvent être utilisés : l'injection hydrodynamique et l'injection électrocinétique. L'introduction de quelques nanolitres dans un capillaire peut se faire très simplement par gravité : c'est l'injection hydrodynamique. Elle est réalisée par application d'une pression pendant un temps défini sur le flacon porte-échantillon dans lequel le capillaire est plongé, ou par création d'une dépression à l'extrémité opposée du capillaire. La vitesse d'injection selon ce procédé est de $5,3 \mathrm{~nL} . \mathrm{s}^{-1}$ dans un capillaire de $50 \mathrm{~cm} \mathrm{x}$ $75 \mu \mathrm{m}$. Ce mode d'injection est le plus couramment utilisé dans les applications toxicologiques. On a décrit des temps d'injection variant de $1 \mathrm{~s}(10)$ à $30 \mathrm{~s}(61,62)$, avec un temps moyen classique de 5 à $10 \mathrm{~s}$ et une pression à l'injection de l'ordre de 0,5 psi $(15,23,29,30)$. Des temps d'injection plus longs produisent un effet d'amplification du champ électrique appelé large volume sample stacking (LVSS) permettant d'améliorer la limite de détection. Dans ce cas, l'injection hydrodynamique nécessite un échantillon sans électrolytes. Des extractions très sélectives sont alors nécessaires et le LVSS est plus fréquemment appliqué à des molécules en solution qu'à des échantillons biologiques. McGrath (61) compare les limites de détection pour la narcotine, le clenbuterol, le flurazépam, la codéine et la péthidine après injections hydrodynamiques pendant un temps de $3 \mathrm{~s}$ et de $30 \mathrm{~s}$ (mode LVSS). Une meilleure sensibilité est obtenue pour le temps d'injection le plus long, avec 
cependant une perte d'efficacité (estimée en nombre de plateaux théoriques). McClean (62) réalise des LVSS avec des temps de 30 à $300 \mathrm{~s}$. Cette technique d'amplification lui permet de détecter des traces de métaux (cobalt, cuivre, plomb, zinc, fer) dans des solutions standards. L'application de cette technique pour détecter des faibles concentrations (analyse de cheveux, par exemple) est restée sans succès en raison d'intensités de courants électriques trop importantes incompatibles avec l'analyse en EC.

En mode d'injection électrocinétique, l'échantillon est introduit dans le capillaire en appliquant une tension électrique pendant un temps donné. Elle est plus faible que celle utilisée pour la séparation, de l'ordre de $10 \mathrm{kV}(21,44,49)$. Des temps de mise sous tension de $10 \mathrm{~s}$ sont très classiquement utilisés, pouvant atteindre $50 \mathrm{~s}$ pour l'analyse du clenbuterol dans les urines (21). Les résultats obtenus sont moins reproductibles pour ce mode d'injection qui présente un caractère très sélectif variant en fonction de facteurs souvent difficiles à contrôler (température à l'injection, composition des solvants injectés, volume total dans le flacon échantillon).

Pour chaque mode d'injection, il existe des méthodes particulières pour concentrer l'échantillon pendant la phase d'injection. La plus classique a pour principe la différence de conductivité existant entre l'échantillon et la solution d'électrolytes : faible concentration en sels dans l'échantillon opposée à une plus forte concentration dans la solution (51). Utilisant le même principe de différence de conductivité, une autre technique d'amplification de champ est réalisée en mode d'injection électrocinétique par la pré-injection d'un bouchon d'eau ou de solvant $(12,51,63)$. Cette demière technique est appelée amplification du champ électrique dans l'échantillon en tête de colonne ou head-column field-amplified sample stacking. Tagliaro (51) améliore le rapport signal/bruit de fond et l'efficacité (estimée en nombre de plateaux théoriques) en injectant avant la solution échantillon contenant de la MDMA, une solution d'acide phosphorique ( $1 \mathrm{mM})$.

En conclusion, une meilleure reproductibilité et l'obtention d'une bonne sensibilité font que l'injection en mode hydrodynamique est très largement utilisée à l'heure actuelle. Taylor (64) a comparé la précision et la sensibilité des deux modes d'injection pour l'analyse des opiacés dans les urines, confirmant la meilleure reproductibilité de l'injection hydrodynamique, et la meilleure sensibilité obtenue en mode d'injection électrocinétique. Les applications de l'injection électrocinétique sont encore limitées à la recherche de certaines classes de molécules (amphétamines, opiacés, cocaïne, LSD) $(12,44,49,51)$ (voir tableau I).

\subsection{4 - Modes de détection}

De nombreux modes de détection sont utilisés en EC : la spectrophotométrie dans l'ultraviolet et le visible, la fluorimétrie, les méthodes électrochimiques et la spectrométrie de masse (SM).

La spectrophotométrie et la fluorimétrie sont les plus fréquemment utilisées en toxicologie. Les premières applications ont surtout été décrites en utilisant un détecteur UV ou un détecteur à barrette de diodes (DBD) qui montrent cependant des limites en terme de sensibilité $(4,7,8,21,22,29,31,33,36,37,40,43,45$, $50,51,58,60,62,65-70)$. Le plus souvent, les limites de détection sont comprises entre $10^{-4}$ et $10^{-6} \mathrm{~mol} / \mathrm{L}$, pour un volume injecté de l'ordre de $10 \mathrm{~nL}$.

Le second mode de détection le plus couramment utilisé en toxicologie est la fluorescence induite par laser (FIL) $(10,15,44,48,49,56)$. Les limites de détection obtenues sont diminuées d'un facteur 100 environ par rapport à la spectrophotométrie (26). Cependant, ce mode de détection est beaucoup plus sélectif et la formation d'un fluorophore est souvent nécessaire pour permettre la détection. D'autre part, l'appareillage est plus complexe, et les longueurs d'ondes d'excitation sont limitées (325 nm pour le laser He-Cd, $488 \mathrm{~nm}$ pour le laser Ar).

Plus récemment, des détections en SM et en SM-SM ont été utilisées en EC $(28,41,47)$. La source d'ionisation de type électrospray permet le couplage EC-SM. Cependant, le choix d'un tampon de migration compatible avec la source d'ionisation reste un problème important. Les tampons de migration les plus couramment utilisés en EC, contenant des phosphates ou des borates non volatils, ne sont pas compatibles avec l'électrospray et les optiques électroniques de la SM. Ainsi, Unger (47) adapte l'analyse en EC-SM d'alcaloïdes en utilisant un tampon d'acétate d'ammonium $(\mathrm{pH} 3,1)$ et acétonitrile $(50 / 50, \mathrm{v} / \mathrm{v})$. Malheureusement, l'augmentation de la force ionique, qui permet d'améliorer les performances de l'EC, n'est pas compatible avec la production d'ions en mode électrospray. La CECM est difficilement compatible à la SM en raison de la nature non volatile des surfactants contenus dans le tampon de migration (71).

Eu égard aux très faibles volumes injectés, les détecteurs doivent être miniaturisés à l'échelle du capillaire : les cellules de détection doivent avoir des volumes de l'ordre du nanolitre. Pour répondre à ces exigences, une petite portion du capillaire lui-même sert généralement 
de cellule de détection. On parle alors de détection en ligne. Comparés au spectrophotomètre et au fluorimètre, le conductimètre et le spectromètre de masse couplés à un appareil d'EC permettent une augmentation de la sensibilité et offrent un complément d'informations, mais présentent l'inconvénient d'être des détecteurs hors ligne, souvent à l'origine d'une perte d'efficacité (72).

La détection spectrophotométrique indirecte est en plein développement pour les molécules qui ne sont pas ou pratiquement pas détectables en UV ou en fluorimètrie. Elle est notamment utilisée dans l'analyse d'anions et de cations inorganiques (73). Dans le cadre de l'analyse en toxicologie, elle permet la détection des ions ammonium, calcium, potassium, magnésium, sodium, acétate, chlorure, citrate, phosphate, sulfate et tartrate dans l'héroöne (74).

\section{4 - Traitement des données et valida- tion des résultats}

La quantification peut être réalisée pour chaque constituant en utilisant la surface corrigée définie par la surface du pic divisée par le temps de migration $\left(t_{M}\right)$. La correction par le temps est apportée afin d'éliminer l'erreur liée aux vitesses de migration différentes dans la cellule de détection. En effet, les molécules dont les $t_{M}$ sont les plus grands restent plus longtemps dans la cellule de détection et donnent donc des surfaces de pics plus importantes. Il est donc primordial de tenir compte de cette correction pour toute quantification.

Le FEO et par conséquent la mobilité des molécules peuvent varier entre deux analyses. L'adsorption et les modifications de la surface du capillaire sont à l'origine de ces variations. Il n'est pas alors toujours suffisant d'utiliser les surfaces corrigées et il est préférable d'injecter des standards ou mieux encore des substances non chargées appelées marqueurs neutres qui ont la particularité de migrer en même temps que le FEO. Les coefficients de variation des $\mathbf{t}_{M}$ sont en général inférieurs à $2 \%(8,18,29,32,33,39,55)$.

Les coefficients de variation de la répétabilité et de la reproductibilité peuvent varier respectivement de 2 à $8 \%$ et 3 à $12 \%(18,44,65,67)$. Les nombreux facteurs pouvant influencer ces valeurs ont été étudiés par Watzig (71) : stabilité de la température pendant l'injection influençant la viscosité du solvant d'injection, électrolyse du tampon de migration avec acidification à l'anode à l'origine de modifications de $t_{M}$, meilleure reproductibilité des résultats obtenus en injection hydrodynamique, importance du pouvoir tampon des phases de migration. La répétabilité des $t_{M}$ est également très dépendante des étapes de conditionnement du capillaire entre chaque analyse. Ces étapes doivent donc être optimisées pour chaque étude (75).

La quantité d'échantillon injectée a une influence importante sur la précision. Un rapport signal sur bruit de fond élevé minimise les erreurs d'intégration et améliore la reproductibilité. Pour augmenter ce rapport, on peut augmenter la concentration de l'échantillon ou augmenter le temps d'injection. Cependant, des temps d'injection allongés compromettent l'efficacité de la séparation (71).

Les limites de détection et de quantification obtenues en EC sont moins bonnes qu'avec la plupart des techniques chromatographiques. Plusieurs exemples décrits dans le paragraphe 3 le montrent. Les facteurs contribuant à abaisser ces limites sont l'augmentation du diamètre du capillaire (qui a pour effet d'augmenter le parcours optique et le volume injecté), l'augmentation du temps ou de la pression d'injection, l'utilisation d'un solvant de l'échantillon de faible conductivité par rapport à celle de l'électrolyte, le choix d'un tampon dont les ions ont une mobilité voisine de l'analyte, l'utilisation de cellules en forme de bulle ou de Z (42).

Un des grands avantages de la technique d'EC est son efficacité. En comparant le nombre de plateaux théoriques des différentes techniques, l'EC se place devant la plupart des techniques chromatographiques avec en moyenne une valeur de l'ordre de 250000 à 500000 , comparé au chiffre de 40000 plus couramment rencontré en CLHP. La simple diminution de la force ionique de l'échantillon (décrite dans le paragraphe 2.3.3) améliore encore cette efficacité.

\section{3 - Applications en toxico- logie clinique et médico- légale}

L'EC apparaît donc comme une technique dont les performances sont suffisamment bonnes pour que des applications en toxicologie clinique ou médico-légale soient envisagées. De nombreuses études ont comparé les résultats obtenus en EC à ceux obtenus avec les techniques les plus performantes CLHP-DBD et CGSM $(23,40,49,76)$.

Le sang et les urines sont deux milieux de choix pour l'analyse toxicologique en EC. Les applications sur d'autres milieux biologiques comme le cheveu, la salive ou le vitré sont encore rares. A notre connaissance, la première équipe et pratiquement la seule ayant appli- 
qué l'EC au dosage de xénobiotiques dans les cheveux est celle de Tagliaro $(8,18,51,65,77)$.

Depuis 1995, l'EC est également employée avec succès pour l'analyse qualitative et quantitative de substances illicites saisies $(31,32,34,54,56,78)$.

\section{1 - Analyse de prélèvements biolo- giques}

L'EC offre de nombreux avantages convenant à l'analyse d'échantillons biologiques : l'utilisation de faibles volumes de prélèvements, la possibilité de répéter plusieurs fois l'analyse sur le même échantillon, la faible consommation de réactifs, les bonnes relations entre la sensibilité et les concentrations dans les milieux biologiques (en particulier avec la FIL), la sensibilité en terme de masse élevée (même dans le cas des détecteurs UV), l'excellente sélectivité et le couplage très satisfaisant avec la SM. L'EC offre également la possibilité d'être utilisée en mode ECZ ou CECM avec le même appareillage, en modifiant uniquement la composition du tampon de migration. On note cependant dans certains cas, une sensibilité limitée en terme de concentration, due à la petite taille de la cellule de détection et au volume d'injection très faible.

Le tableau I rassemble des exemples d'analyses de xénobiotiques dans les milieux biologiques en EC décrits dans la littérature.

\subsection{1 - Sang et urine}

Wernly et Thormann (5) furent les premiers à décrire en CECM l'analyse dans les urines de différents xénobiotiques : benzoylecgonine, morphine, héroïne, 6MAM, méthamphétamine, codéine, amphétamine, cocaïne, méthadone et méthaqualone. Le seuil de détection de l'ordre de $100 \mathrm{ng} / \mathrm{mL}$ était comparable à ceux de l'immunoanalyse pour les mêmes substances et l'EC a été ainsi proposée pour confirmer des dépistages positifs. Dans des conditions similaires, l'analyse de benzodiazépines a été réalisée dans les urines (66). Malgré le manque de sensibilité de l'ECZ, l'utilisation de la CECM a permis le dosage de l'acide 11-nor- $\Delta-9$ THC-COOH, métabolite urinaire majeur du $\Delta-9-\mathrm{THC}$ (7) : un seuil de détection de l'ordre de $10 \mathrm{ng} / \mathrm{mL}$ était obtenu.

L'ECZ permet également le dosage urinaire de la méthadone et de son principal métabolite, l'EDDP (voir tableau I). Les temps de migration sont inférieurs à 10 minutes et la limite de détection est de l'ordre de $20 \mathrm{ng} / \mathrm{mL}$ (36). La stéréosélectivité du métabolisme de la méthadone dans les urines a été étudiée en utilisant comme sélecteurs chiraux des cyclodextrines diluées dans le tampon de migration (16).

En 1996, Taylor (64) utilise l'ECZ pour la détermination d'opiacés dans les urines. Les auteurs améliorent la sensibilité de la technique en utilisant un mode d'injection de type électrocinétique et une amplification du champ électrique après extraction des échantillons urinaires en phase solide : la limite de détection est située entre 4 et $9 \mathrm{ng} / \mathrm{mL}$.

De nombreuses études sont décrites sur le plasma ou sur l'urine pour les dérivés amphétaminiques et analogues $(22,37)$, la méthaqualone $(58)$, le $\operatorname{LSD}(44,49)$, l'acide acétylsalicylique et ses métabolites (79), le paracétamol et ses métabolites (70), ainsi que dans les urines pour la méthadone (40), les analgésiques non opioides (28), les benzodiazépines (80) (voir tableau I). Hudson en 1998 (50) développe une méthode de screening dans le sang de molécules d'intérêt médico-légal. Un capillaire de $50 \mu \mathrm{m}$ (d.i) et de $60 \mathrm{~cm}(l)$ et une détection en mode DBD sont utilisés. Deux types d'extraction sont réalisés : une extraction en phase liquide permettant d'extraire les molécules à caractère basique et une extraction en phase solide pour les molécules à caractère acide. Les extraits sont injectés en mode électrocinétique $(8 \mathrm{~s}, 10 \mathrm{kV})$ et hydrodynamique $(8 \mathrm{~s}, 0,5$ psi). Deux tampons sont employés : les molécules à caractère basique sont analysés avec un tampon phos-

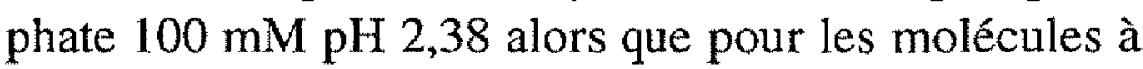
caractère acide un tampon borate $100 \mathrm{mM} \mathrm{pH} \mathrm{8,5} \mathrm{est}$ utilisé. Les temps de migration relatifs ainsi que les mobilités électrophorétiques ont été obtenus pour 650 molécules.

Comparée à celle de la CG-SM et de la CLHP, la sensibilité de l'EC reste encore modeste. Cependant, pour la plupart des molécules analysées, une séparation classique en mode d'injection hydrodynamique à partir de $5 \mathrm{~mL}$ d'urine permet de détecter des concentrations de l'ordre de $50 \mathrm{ng} / \mathrm{mL}$, nettement inférieures aux seuils de positivité des techniques immunologiques utilisées dans les dépistages urinaires (25). Frost (44) dose le LSD dans les urines et le plasma avec une limite de détection de 0,1 à $0,2 \mathrm{ng} / \mathrm{mL}$ en mode FIL après une injection électrocinétique. D'autres exemples décrits dans le tableau I $(12,22)$ montrent l'amélioration de la sensibilité par l'utilisation de techniques d'injection électrocinétique.

La combinaison de l'immunoanalyse et de l'EC constitue un nouveau concept pour le dépistage et le dosage de plusieurs molécules dans les urines en une opération, sans passer par l'étape d'extraction des xénobio- 
tiques. Elle allie la rapidité et la simplicité de l'EC à l'utilisation de réactifs d'immunochimie largement distribués dans le commerce et éprouvés depuis longtemps $(10,41)$. Le principe est le suivant : 20 à $50 \mu \mathrm{L}$ d'urine contenant le ou les xénobiotiques à doser sont incubés avec les mêmes volumes d'anticorps froids et d'antigènes marqués avec un fluorophore. Une aliquote du mélange est injectée dans le système d'EC. Les complexes antigènes marqués-anticorps et les antigènes marqués restés libres sont suffisamment séparés pour pouvoir être détectés par FIL. Caslavska (10) vient de montrer que les limites de détection étaient de l'ordre de $30 \mathrm{ng} / \mathrm{mL}$ pour la morphine, l'amphétamine, la benzoylecgonine et la méthadone. Ses résultats avaient été validés par CG-SM. Des études de faisabilité ont déjà été réalisées avec succès pour le dosage de médicaments (digoxine, méthadone, théophylline, cyclosporine) et stupéfiants (phéncyclidine, 11-nor- $\Delta 9$-THC, benzoylecgonine, amphétamine et méthamphétamine) (10). Le détecteur est alors fonction des réactifs d'immunoanalyse utilisés : laser He-Ne ou laser Ar pour la FPIA, spectrophotomètre pour l'EMIT ou l'immunoaffinité. Cette association de l'immunochimie à l'EC permet d'obtenir une technique simple, rapide, sensible, présentant cependant les inconvénients de non spécificité des techniques immunologiques. La fabrication et l'utilisation de ses propres anticorps et antigènes marqués permettra cependant de multiplier les possibilités de cette méthodologie.

Avec l'utilisation de sélecteurs chiraux dans le tampon de migration, l'EC s'est énormément développée. Elle est devenue une technique simple et peu coûteuse pour la séparation et l'analyse des énantiomères. Cette approche est devenue très intéressante en toxicologie notamment dans les contrôles antidopage ou dans l'étude du métabolisme des médicaments et des drogues. On citera par exemple, l'analyse des énantiomères de la méthadone, de l'amphétamine, de la méthamphétamine, de l'éphédrine, de la MDMA, de la MDA et de la MDEA dans les urines (16-18, 22, 37, 59), du thiopental ou du pentobarbital dans le plasma (11) et de la zopiclone et de ses métabolites dans les urines (15). Ces approches sont simples, reproductibles et peu d'interférences sont décrites avec les substances de nature basique classiquement rencontrées dans les urines.

Le nombre d'études utilisant le couplage de l'EC et de la SM pour l'analyse de toxiques dans les milieux biologiques est en forte progression. On pourra citer la recherche de différents analgésiques et anti-inflammatoires et de leurs métabolites (paracétamol, acide acétylsalicylique, antipyrine, ibuprofène, naproxène, kéto- profène et propyphénazone) (28) ou de la méthadone (41). Plus récemment, l'EC-SM-SM a été employée pour identifier la méthadone et son métabolite principal, l'EDDP, dans les urines (41). Cependant, les détecteurs optiques restent encore les plus utilisés.

Le tableau I montre bien que toutes les études décrites ont été réalisées sur des capillaires en silice fondue et non traités. La plupart des analyses utilisent un tampon aqueux avec ou sans modificateur organique. En début de l'année 2000, nous n'avons retrouvé qu'une étude utilisant l'EC en milieu non aqueux, pour l'analyse en mode de détection UV de l'acide acétylsalicylique et de ses métabolites après extraction solide-liquide à partir d'urines et de plasma (79).

\subsection{2 - Cheveux}

L'analyse toxicologique des cheveux est devenu le principal outil d'investigation du passé, de l'exposition temporaire à l'utilisation chronique de substances illicites (81).

Les premières expériences en ECZ en mode de détection UV donnent de bons résultats en terme de sélectivité et d'efficacité ( $>350000$ plateaux théoriques) pour l'analyse de la cocaïne et de la morphine dans les cheveux, mais limités en terme de sensibilité $\quad(<$ $0,2 \mathrm{ng} / \mathrm{mg}$ ) pour $100 \mathrm{mg}$ d'échantillon (65). Les très faibles teneurs en xénobiotiques rencontrées habituellement dans les cheveux ont conduit à trouver des solutions pour améliorer ce problème de sensibilité. La plus simple est l'utilisation de capillaires de diamètre légèrement supérieur $(75 \mu \mathrm{m}$ au lieu de $50 \mu \mathrm{m})(8)$. A partir de 1995, différentes techniques d'amplification de champ électrique dans la zone d'injection sont développées pour l'analyse du cheveu. Elles permettent la concentration de l'échantillon et par conséquent l'augmentation de la sensibilité sans perte d'efficacité (8, 51). Pour réaliser cet effet d'amplification, les échantillons peuvent être injectés dilués dans des solvants de faible conductivité comme de l'eau distillée (8). Mais il s'avère que cette première technique permettant d'augmenter de l'ordre de cinq fois la sensibilité, n'est pas suffisante pour une application sur les cheveux d'utilisateurs occasionnels de ce type de substances (héroïne, cocaïne). En 1998, Tagliaro (51) diminue les limites de détection en utilisant la technique d'amplification de champ électrique dans l'échantillon en tête de colonne. Il réalise successivement avant l'injection de l'échantillon, un rinçage à l'eau de la cuve contenant l'extrémité du capillaire du coté de l'anode pendant $5 \mathrm{~s}$ et une préinjection de solvant de faible conductivité (acide 
phosphorique $0,1 \mathrm{mM}$ ) pendant $10 \mathrm{~s}$ à 0,5 psi. Dans ces conditions, en mode de détection UV, les limites de détection sont de $2 \mathrm{ng} / \mathrm{mL}$ pour la MDMA et la MDE, de $3 \mathrm{ng} / \mathrm{mL}$ pour la MDA, de $8 \mathrm{ng} / \mathrm{mL}$ pour la cocaïne et de $6 \mathrm{ng} / \mathrm{mL}$ pour la morphine. Il arrive ainsi à mettre en évidence des concentrations de stupéfiants de 0,01 à $0,02 \mathrm{ng} / \mathrm{mg}$ de cheveux (51).

L'utilisation de la CECM a également montré son intérêt pour la recherche de cocaine et morphine dans les cheveux (8). La sensibilité est moins bonne mais la CECM permet de travailler avec des volumes de reprise d'échantillons plus élevés (100 à $200 \mu \mathrm{L}$ au lieu de $20 \mu \mathrm{L}$ en ECZ) assurant ainsi une meilleure reproductibilité des résultats (8).

Ainsi, le couplage de l'électrophorèse et de la technique d'amplification de champ électrique dans l'échantillon en tête de colonne peut contribuer à la banalisation de l'EC pour l'analyse toxicologique des cheveux. Cependant, la sensibilité reste modérée en terme de concentration (de l'ordre $\mathrm{du} \mathrm{ng} / \mathrm{mL}$ ) en raison d'un volume d'injection très faible et de la petite taille de la cellule de détection.

\subsection{3 - Autres prélèvements}

D'autres milieux ont été également étudiés. Hortin a montré l'intérêt de l'utilisation de l'EC pour l'analyse du contenu gastrique et de la bile (52). Il a dosé l'azide de sodium après piégeage de celui-ci par de la soude dans une cellule de Conway. Avec une détection spectrophotométrique, la limite de quantification était de 20 $\mu \mathrm{g} / \mathrm{mL}$, ce qui était amplement suffisant pour le contenu gastrique. En mode CECM, l'injection directe du contenu gastrique est possible après simple filtration, les protéines étant dénaturées par le détergent du tampon de migration. Ainsi, Plaut (58) a pu doser la méthaqualone à des taux supérieurs à $30 \mu \mathrm{g} / \mathrm{mL}$. Il remarque cependant qu'une extraction liquide-liquide préalable permet d'abaisser ce seuil d'un facteur 10. Pourtant, l'avantage de l'EC tient dans ce que, même si le contenu gastrique a un aspect solide, le volume de phase liquide nécessaire est si faible qu'il sera toujours possible d'en récupérer une petite fraction par centrifugation. L'inconvénient de la matrice complexe que constitue le contenu gastrique peut être diminué par dilution dans de l'eau, ce qui diminue la force ionique de l'échantillon.

Dans un domaine proche de la toxicologie, Ferslew (30) a montré qu'il était possible de doser simultanément plusieurs électrolytes dans le vitré de l'œil (potassium, sodium, calcium et lithium). Il procède à une injection directe de l'échantillon dilué au $1 / 100^{\mathrm{ems}}$ dans de l'eau. Bien qu'utilisant un électrolyte complexe contenant de l'acide hydroxybutyrique, un chélateur et de la 4-éthylbenzylamine, avec une détection spectrophotométrique inverse, cette méthode est un bon exemple des développements possibles de l'EC.

\section{2 - Analyse de substances illicites sai- sies}

Le domaine d'investigation de l'EC en toxicologie s'est étendu à l'analyse qualitative et quantitative des substances illicites saisies. De nombreux exemples sont décrits en ECZ sans additifs dans le tampon de migration (32-34, 46, 47, 82), et en CECM $(3,31,54,56)$. Des études plus rares ont été réalisées en milieu non aqueux $(83)$, en $\operatorname{CEC}(84,85)$ ou sur des capillaires recouverts de polymères (86). L'analyse chirale par complexation avec des cyclodextrines s'est aussi considérablement développée $(45,54)$.

Des méthodes en ECZ ont ainsi été élaborées et validées pour l'amphétamine et les phénéthylamines (32, 33,82 ), la psilocybine (46), la cocaïne (34) et des a!raloïdes indoliques monoterpéniques, benzophénanthridiniques, isoquinoliniques de l'opium et dérivés de la $\beta$-carboline (47). L'EC présente ici des avantages sur les techniques chromatographiques : pas de dérivation des dérivés amphétaminiques ou pas de dénaturation de la psilocybine sous l'influence de la chaleur. Pour les analyses de la cocaïne par exemple, la faible consommation de solvants, la quantité minime d'échantillon nécessaire, les temps d'analyse très courts sont des potentiels intéressants pour des laboratoires à très forte activité. Ainsi, Krawczeniuk (34) montre que la cocaïne, la plupart des adultérants et des impuretés classiques sont séparés en moins de 9 minutes. Seules la benzocaine (adultérant) et la benzoylecgonine (impureté) migrent avec le FEO. La linéarité de la méthode pour le dosage de la cocaïne est décrite entre 0,014 à $1,017 \mathrm{mg} / \mathrm{mL}$ en mode DBD. La technique est en accord avec les résultats quantitatifs obtenus en CLHP et CG-SM (34).

La SM couplée à l'EC offre la meilleur spécificité de toute les détections. Elle est particulièrement avantageuse pour l'analyse de substances plus difficiles à analyser en CG-SM comme certains opioïdes, les benzodiazépines, le LSD et la psilocybine. Unger (47) a largement montré l'intérêt de l'utilisation de l'EC-SM dans l'identification d'alcaloïdes (voir tableau III).

Weinberger et Lurie en 1991 (3) utilisèrent les premiers la CECM pour l'analyse de substances illicites. Depuis, 
quelques études ont été décrites en mode micellaire pour l'analyse de saisies d'héroïne, d'opium, de cocaïne, d'amphétamines, de LSD et de stéroïdes anabolisants. Elles sont résumées dans la revue de Lurie (54). Un exemple d'analyse d'amphétamines est détaillé dans le tableau III (31). Cependant, ces études restent peu nombreuses et l'utilisation préférentielle de l'ECZ est certainement due à la simplicité de cette technique.

L'EC en milieu non aqueux, caractérisée par de faibles courants d'analyse et par la possibilité d'utiliser des solvants volatils, est une technique bien adaptée à l'analyse des substances de nature basique. Bjornsdottir (83) décrit la séparation de 16 substances pouvant être trouvées dans des préparations illicites (voir tableau III). Il réalise une injection à la cathode (électrode de détection) en polarité inversée, mode d'injection particulièrement intéressant pour des temps d'analyse très courts : les seize molécules sont séparées en moins de 2 minutes.

La CEC apparaît également adaptée à l'analyse de substances illicites quelle que soit leur nature $(25,84,85)$. De longues colonnes, des particules de faible taille génèrent une efficacité élevée. L'utilisation de la CEC est décrite pour l'analyse de substances basiques dans le tableau III (84). L'intérêt de cette approche est de pouvoir solubiliser dans une même phase d'injection des molécules hydrophiles et hydrophobes compatibles avec le système de séparation. Cette combinaison de techniques chromatographiques et électrophorétiques est à l'origine de sélectivités différentes exploitées pour les séparations les plus difficiles (25).

La reconnaissance et l'analyse centésimale des isomères optiques dans des substances illicites peut permettre l'identification de la voie de synthèse, la comparaison entre eux des différents lots saisis et indiquer la source de la saisie (87). Le rôle de l'EC dans la détermination des formes énantiomèriques devient prépondérant $(20$, 45,60 ). Lurie (60) étudie l'effet de sélecteurs chiraux anioniques sur la CEC d'un mélange chiral de phényléthylamines et d'impuretés neutres non chirales présents dans de la méthamphétamine illicite. En utilisant une sulfobutyl(VII)-ether- $\beta$-cyclodextrine, la séparation est réalisée en $27 \mathrm{~min}$, les phenéthylamines étant séparées en moins de $10 \mathrm{~min}$ (voir tableau III).

\section{4 - Conclusion}

L'EC est un outil permettant de résoudre de nombreux problèmes analytiques. Elle est maintenant utilisable en routine dans un laboratoire de toxicologie, si l'on garde à l'esprit quelques points fondamentaux : la précision du $\mathrm{pH}$ des solutions de travail est primordiale ; le capillaire doit être considéré comme un réactif sujet à des variations physiques et chimiques ; l'EC est une nanométhode, donc sensible aux moindres variations de pression et aux impuretés des réactifs ; les électrolytes et les xénobiotiques ne sont mis en mouvement que grâce à un champ électrique dont les moindres variations vont influencer la répétabilité des analyses $(25,88-90)$.

En toxicologie analytique, les modes de détection les plus utilisés sont l'UV et la FL, dont la sensibilité reste moyenne. Les progrès récents des couplages EC-SM et EC-SM-SM permettent de l'augmenter considérablement. On pourra donc bientôt tirer complètement avantage de la possibilité d'injecter directement l'échantillon biologique, évitant la phase d'extraction.

L'utilisation de séparateurs chiraux a permis de résoudre des problèmes difficiles dans l'analyse des énantiomères, notamment pour les phénéthylamines.

L'EC semble donc promise à un avenir intéressant dans le domaine de la toxicologie clinique et médico-légale, mais il reste beaucoup à explorer.

\section{Références}

1. Hjerten S., Free solution electrophoresis in $3 \mathrm{~mm}$ tube. Chomatogr. Rev. $1967 ; 9: 122-219$.

2. Jorgenson J., Lukacs K., High-resolution separations based on electrophoresis and electroosmosis. J. Chromatogr. $1981 ; 218: 209-16$.

3. Weinberger R., Lurie I., Micellar electrokinetic capillary chromatography of illicit drug substances. Anal. Chem. $1991 ; 63: 823-7$.

4. Thormann W., Meier P., Marcolli C., Binder F., Analysis of barbiturates in human serum and urine by high-performance capillary electrophoresis-micellar electrokinetic capillary chromatography with on-column multi-wavelength detection. J. Chromatogr. $1991 ; 545: 445-60$.

5. Wernly P., Thormann W., Analysis of illicit drugs in human urine by micellar electrokinetic capillary chromatography with on-column fast scanning polychrome absorption detection. Anal. Chem. 1991 ; $63: 2878-82$.

6. Wernly P., Thormann W., Drug of abuse confirmation in human urine using stepwise solid-phase extraction and micellar electrokinetic capillary chromatography. Anal. Chem. $1992 ; 64: 2155-9$.

7. Wernly P., Thormann W., Confirmation of 11-nor- $\Delta 9$ tetrahydrocannabinol-9-carboxylic acid in urine with micellar electrokinetic capillary chromatography. J. Chromatogr. $1992 ; 608: 251-6$.

8. Tagliaro F., Smith F.P., Turrina S., Deyl Z., Marigo M., Capillary electrophoresis : a new tool in forensic toxicology. Applications and prospects in hair analysis for illicit drugs. Forensic Sci. Int. $1995 ; 70: 93-104$. 
9. Tagliaro F., Smith F., Forensic capillary electrophoresis. Trends Anal. Chem. $1996 ; 15,513-25$.

10. Caslavska J., Alleman D., Thormann W., Analysis of urinary drugs of abuse by a multianalyte capillary electrophoretic immunoassay. J. Chromatogr. A $1999 ; 838$ (1-2): 197-211.

11. Zaugg S., Caslavska J., Theurillat R,, Thormann W, Characterization of the stereoselective metabolism of thiopental and its metabolite pentobarbital via analysis of their enantiomers in human plasma by capillary electrophoresis. J. Chromatogr. A 1999 ; 838 (1-2) : 237-49.

12. Wey A., Zhang C., Thormann W., Head-column fieldamplified sample stacking in binary system capillary electrophoresis. Preparation of extracts for determination of opioids in microliter amounts of body fluids. J. Chromatogr. A $1999 ; 853$ (1-2): 95-106.

13. Lurie I., Klein R., Dal Cason T., LeBelle M., Brenneisen R., Weinberger R., Chiral resolution of cationic drugs of forensic interest by capillary electrophoresis with mixtures of neutral and anionic cyclodextrins. Anal. Chem. $1994 ; 66(22): 4019-26$.

14. Fanali S., Identification of chiral drug isomers by capillary electrophoresis. J. Chromatogr. $1996 ; 735: 77-121$.

15. Hempel G., Blaschke G., Enantioselective determination of zopiclone and its metabolites in urine by capillary electrophoresis. J. Chromatogr. B Biomed. Appl. 1996; 675 (1) : 139-46.

16. Lanz M., Thormann W., Characterization of the stereoselective metabolism of methadone and its primary metabolite via cyclodextrin capillary electrophoretic determination of their urinary enantiomers. Electrophoresis $1996 ; 17(12): 1945-9$.

17. Frost M., Köhler H., Blaschke G., Enantioselective determination of methadone and its main metabolite 2-ethylidene-1,5-dimethyl-3,3-diphenylpyrrolidine (EDDP) in serum, urine and hair by capillary electrophoresis. Electrophoresis $1997 ; 18$ (6) : 1036-34.

18. Scarcella D., Tagliaro F., Turrina S., Manetto G., Nakahara Y., Smith FP., Marigo M., Optimization of a simple method for the chiral separation of phenethylamines of forensic interest based on cyclodextrin complexation capillary electrophoresis and its preliminary application to the analysis of human urine and hair. Forensic Sci. Int. 1997 ; 89 : 33-46.

19. Tagliaro F., Manetto G., Bellini S., Pisi P., Scarcella D., Turrina S., Marigo M., Capillary electrophoresis, a first choice technique for the chiral analysis of illicit drugs. Application to analysis of amphetamine and congeners. XXXV TIAFT, Padova, Italy, 24-28 Février 1997.

20. Varesio E., Gauvrit J., Longeray R., Lanteri P., Veuthey $J$., Central composite design in the chiral analysis of amphetamines by. capillary electrophoresis. Electrophoresis $1997 ; 18(6)$ : $931-7$.

21. Gausepohl C., Blaschke G., Stereoselective determination of clenbuterol in human urine by capillary electrophoresis. J. Chromatogr. B Biomed. Sci. Appl. 1998 ; 713 (2) : 443-6.

22. Tagliaro F., Manetto G., Bellini S., Scarcella D., Smith F, Marigo M., Simultaneous chiral separation of 3,4methylenedioxymetamphetamine, 3,4-methylenedioxy- amphetamine, 3,4-methylenedioxyethylamphetamine, ephedrine, amphetamine and methamphetamine by capillary electrophoresis in uncoated and coated capillaries with native beta-cyclodextrin as the chiral selector : preliminary application to the analysis of urine and hair. Electrophoresis $1998 ; 19$ (1) : 42-50.

23. Iwanicki R., Maier K., Zlonick J., Liu R., Kuo T., Tagliaro F, Separation of enantiomeric ephedrine and pseudoephedrine-high pressure liquid chromatography and capillary electrophoresis. J. Forensic Sci. $1999 ; 44$ (3) : 470-4.

24. Veuthey JL., Rudaz S., Chiralité en électrophorèse capillaire et en chromatographie liquide. Forum Labo, 28-31 Mars 2000, Paris.

25. Thormann W., Wey A., Lurie I., Gerber H., Byland C., Malik N., Hochmeister M., Gehrig C., Capillary electrophoresis in clinical and forensic analysis : recent advances and breakthrough to routine applications. Electrophoresis 1999 ; 20 (15-16) : 3203-36.

26. Gareil P., L'électrophorése de zone et la chromatographie électrocinétique capillaires. I - Principes et notions fondamentales. Analusis $1990 ; 18: 221-41$.

27. Blessum C., Jeppsson J., Aguzzi F., Bernon H., Bienvenu J., L'électrophorèse capillaire : principe et applications au laboratoire de biologie clinique. Ann. Biol. Clin. $1999 ; 57: 643-57$.

28. Heitmeier S., Blaschke G., Direct assay of nonopïoid analgesics and their metabolites in human urine by capillary electrophoresis and capillary electrophoresis-mass spectrometry. J. Chromatogr. B Biomed. Sci. Appl. $1999 ; 721$ (1) : 109-25.

29. Hyotylainen T., Siren H., Riekkola M., Determination of morphine analogues, caffeine and amphetamine in biological fluids by capillary electrophoresis with the marker technique. J. Chromatogr. A $1996 ; 735$ (1-2) : 439-47.

30. Ferslew K., Hagardon A., Harrison M., McCormick W., Capillary ion analysis of potassium concentrations in human vitreous humor. Electrophoresis $1998 ; 19: 6-10$.

31. Trenerry V., Robertson J., Wells R., Analysis of illicit amphetamine seizures by capillary electrophoresis. $\mathrm{J}$. Chromatogr. A 1995 ; 708 : 169-76.

32. Frost M., Köhler H., Blaschke G., Analysis of "Ecstasy" by capillary electrophoresis. Int. J. Legal Med. 1996 ; $109: 53-7$.

33. Esseiva P., Lock E., Gueniat O., Cole M., Identification and quantification of amphetamine and analogues by capillary zone electrophoresis. Sci. Justice $1997 ; 37$ (2) : 113-9.

34. Krawczeniuk A., Bravenec V., Quantitative determination of cocaine in illicit powders by free zone capillary electrophoresis. J. Forensic Sci. 1998 ; 43 (4) : 738-43.

35. Lloyd D., Capillary electrophoretic analyses of drugs in body fluids : sample pretreatment and methods for direct injection of biofluids. J. Chromatogr. A $1996 ; 735: 29$ 42.

36. Molteni S., Caslavska J., Allemann D., Thormann W., Determination of methadone and its primary metabolite in human urine by capillary electrophoretic techniques. J. Chromatogr. B Biomed. Appl. 1994 ; 658 (2) : 355-67. 
37. Ramseier A., Caslavska J., Thormann W., Screening for urinary amphetamine and analogs by capillary electrophoretic immunoassays and confirmation by capillary electrophoresis with on-column multiwavelength absorbance detection. Electrophoresis $1998 ; 19$ : 2956-66.

38. Jinno K., Han Y., Sawada H., Analysis of toxic drugs by capillary electrophoresis using polyacrylamide-coated columns. Electrophoresis $1997 ; 18$ (2) : 284-6.

39. Chee G., Wan T., Reproductible and high-speed separation of basic drugs by capillary zone electrophoresis. J. Chromatogr. $1993 ; 612: 172-7$.

40. McAvoy Y., Cole M., Gueniat O., Analysis of amphetamines by supercritical fluid chromatography, high-performance liquid chromatography, gas chromatography and capillary zone electrophoresis ; a preliminary comparison. Forensic Sci. Int. 1999 ; 102 : 13-22.

41. Thormann W., Lanz M., Caslavska J., Siegenthaler P., Portmann R., Screening for urinary methadone by capillary electrophoretic immunoassays and confirmation by capillary electrophoresis-mass spectrometry. Electrophoresis $1998 ; 19: 57-65$.

42. Fabre H., Validation des méthodes d'électrophorèse capillaire appliquées à l'analyse des composés pharmaceutiques. Analusis $1999 ; 27$ (2) : 155-60.

43. Tagliaro F., Antonioli C., Moretto S., Archetti S., Ghielmi S., Marigo M., High-sensitivity low-cost methods for determination of cocaine in hair : high-performance liquid chromatography and capillary electrophoresis. Forensic Sci. Int. 1993 ; 63 : 227-38.

44. Frost M., Köhler H., Blaschke G., Determination of LSD in blood by capillary electrophoresis with laser-induced fluorescence detection. J. Chromatogr. B Biomed. Sci. Appl. $1997 ; 693: 313-9$.

45. Bokor I., Trenerry V., Scheelings P., Separation and quantitation of optical isomers of methylamphetamine samples by capillary electrophoresis. Forensic Sci. Int. $1997 ; 85: 177-92$.

46. Pedersen-Bjergaard S., Sannes E., Rasmussen K., Tonnesen F., Determination of psylocibin in Psicocybe semilanceata by capillary zone electrophoresis. J. Chromatogr. B $1997 ; 694: 375-381$.

47. Unger M., Stöckigt D., Belder D., Stöckigt J., General approach for the analysis of various alkaloid classes using capillary electrophoresis and capillary electrophoresis-mass spectrometry. J. Chromatogr. A 1997; 767 : 263-76.

48. Couderc F, Caussé E., Bayle C., Drug analysis by capillary electrophoresis and laser-induced fluorescence. Electrophoresis $1998 ; 19: 2777-90$.

49. Frost M., Köhler H., Analysis of lysergic acid diethylamide : comparison of capillary electrophoresis with laser-induced fluorescence (CE-LIF) with conventional techniques. Forensic Sci. Int. $1998 ; 92: 213-8$.

50. Hudson J., Golin M., Malcolm M., Whiting C., Capillary zone electrophoresis in a comprehensive screen for drugs of forensic interest in whole blood : an update. Can. Soc. Forens. Sci. J. 1998 ; 31 (1) : 1-29.
51. Tagliaro F, Manetto G., Crivelente F., Scarcella D. Marigo M., Hair analysis for abused drugs by capillary zone electrophoresis with field-amplified sample stacking. Forensic Sci. Int. 1998 ; 92 : 201-11

52. Hortin G., Dey S., Hall M., Robinson C., Detection of azide in forensic samples by capillary electrophoresis. J. Forensic Sci. 1999 ; 44 (6) : 1310-3.

53. Imazawa M., Hatanaka Y., Micellar electrokinetic capillary chromatography of benzodiazepine antiepileptics and their desmethyl metabolites in blood. J. Pharm. Biomed. Anal. $1997 ; 15$ (9-10) : 1503-8.

54. Lurie I., Application of micellar electrokinetic capillary chromatography to the analysis of illicit drug seizures. J. Chromatogr. A $1997 ; 780: 265-84$

55. Tagliaro F., Smith F.P., Turrina S., Equisetto V., Marigo M., Complementary use of capillary zone electrophoresis and micellar electrokinetic capillary chromatography for mutual confirmation of results in forensic drug analysis. J. Chromatogr. A $1996 ; 735: 227-35$.

56. Lurie I., Chan K., Spratley T., Casale J., Issaq H., Separation and detection of acidic and neutral impurities in illicit heroin via capillary electrophoresis. J. Chromatogr. B Biomed. Appl. 1995 ; 669 (1) : 3-13.

57. Spencer B., Zhang W., Purily W., Capillary electrophoretic separation of tricyclic antidepressants using charged carboxymethyl-beta-cyclodextrin as a buffer additive. Electrophoresis $1997 ; 18: 736-44$.

58. Plaut O., Girod C., Staub C., Analysis of methaqualone in biological matrices by micellar electrokinetic capillary chromatography. Comparison with gas chromatography-mass spectrometry. Forensic Sci. Int. $1998 ; 92$ (2-3) : 219-27.

59. Lanz M., Brenneisen R., Thormann W., Enantioselective determination of 3,4-methylene-dioxy methamphetamine and two of its metabolites in human urine by cyclodextrin-modified capillary zone electrophoresis. Electrophoresis $1997 ; 18: 1035-43$.

60. Lurie I., Odeneal II N., McKibben T., Casale J., Effects of various anionic chiral selectors on the capillary electrophoresis separation of chiral phenethylamines and achiral neutral impurities present in illicit methamphetamine. Electrophoresis $1998 ; 19: 2918-25$.

61. McGrath G., Smyth W., Large-volume sample stacking of selected drugs of forensic significance by capillary electrophoresis. J. Chromatogr. B Biomed. Appl. 1996 ; $681: 125-31$.

62. McClean S., O'Kane E., Coulter D., McLean S., Smyth W. Capillary electrophoretic determination of trace metals in hair samples and its comparison with high performance liquid chromatography and atomic adsorption spectrometry techniques. Electrophoresis $1998 ; 19: 11-8$.

63. Zhang C., Thormann W., Head-column field-amplified sample stacking in binary system capillary electrophoresis. 2. Optimization with a preinjection plug and application to micellar electrokinetic chromatography. Anal. Chem. $1998 ; 70$ (3) : 540-8.

64. Taylor R., Low A., Reid R., Determination of opiates in urine by capillary electrophoresis. J. Chromatogr. B. 
Biomed. Appl. 1996 ; 675 (2) : 213-23.

65. Tagliaro F., Poiesi C., Aiello R., Dorizzi R., Ghielmi S., Marigo M., Capillary electrophoresis for the investigation of illicit drugs in hair : determination of cocaïne and morphine. J. Chromatogr. $1993 ; 638$ (2) : 303-9.

66. Schafroth M., Thormann W., Allemann D., Micellar electrokinetic capillary chromatography of benzodiazepines in human urine. Electrophoresis $1994 ; 15: 72-8$.

67. Tomita M., Okuyama T ., Application of capillary electrophoresis to the simultancous screening and quantitation of benzodiazepines. J. Chromatogr. B Biomed. Appl. $1996 ; 678(2): 331-7$.

68. Tagliaro F., Turrina S., Pisi P., Smith F.P., Marigo M., Determination of illicit and/or abused drugs and compounds of forensic interest in biosamples by capillary electrophoretic/electrokinetic methods. J. Chromatogr. B $1998 ; 713: 27-49$.

69. Perrigo B., A report on the use the P/ACETM capillary electrophoresis system as a stand alone UV/VIS spectrophotometer in a forensic toxicology laboratory. Can. Soc. Forens. Sci. J. 1998 ; 31 (4) : 275-84.

70. Bjornsdottir I., Tjornelund J., Hansen S., Nonaqueous capillary electrophoresis - its applicability in the analy. sis of food, pharmaceuticals and biological fluids. Electrophoresis $1998 ; 19$ (12) : 2179-86.

71. Wätzig H., Degenhardt M., Kunkel A., Strategies for capillary electrophoresis : method development and validation for pharmaceutical and biological applications. Electrophoresis $1998 ; 19: 2695-752$.

72. Fillet M., Bechet P., Hubert P., Crommen J., L'électrophorèse capillaire ; Principes, mises en cuvre et applications. STP Pharma Pratiques 1999 ; 9 (3) : 22543.

73. Altria K., Overview of capillary electrophoresis and capillary electrochromatography. J. Chromatogr. A $1999 ; 856(1-2): 443-63$.

74. Lurie I., The analysis of cations and anions in illicit heroin using capillary electrophoresis with indirect UV detection. J. Capillary Electroph. 1996 ; 3 (5) : 237-42.

75. Faller T., Engelhart H., How to achieve higher repeatibility and reproductibility in capillary electrophoresis. $J$. Chromatogr. A $1999 ; 853: 83-94$.

76. Almirall J., Garcia A., Quantitative determination of common benzodiazepines by free zone electrophoresis. $52^{\text {ind }}$ mecting of American Academy of Forensic Sciences (AAFS), February 21-26, 2000, Reno, NV.

77. Tagliaro F., Smith F.P., De Battisti Z., Manetto G., Marigo M., Hair analysis, a novel tool in forensic and biomedical sciences : new chromatographic and electrophoretic/electrokinetic analytical strategies. J. Chromatogr. B Biomed.
Sci. Appl. 1997 ; 689 (1) : 261-71.

78. Tagliaro F., Turrina S., Smith F.P., Capillary electrophoresis : principles and applications in illicit drug analysis. Forensic Sci. Int. 1996 ; 77 : 211-29.

79. Hansen S., Jensen M., Bjornsdottir I., Assay of acetylsalicylic acid and three of its metabolites in human plasma and urine using non-aqueous capillary electrophoresis with reversed electroosmotic flow. J. Pharm. Biomed. Anal. $1998 ; 17(6-7): 1155-60$.

80. Plaut O., Staub C., Analyse de benzodiazépines par électrophorèse capillaire. Toxicorama 1996 ; VIII (3) : 59-66.

81. Kintz P., Matrices alternatives et toxicologie médicolégale. In : Toxicologie et pharmacologie médicolégales. Kintz P., Elsevier, Paris, 1998 ; 685-710.

82. Sadeghipour F., Varesio E., Giroud C., Rivier L., Veuthey J., Analysis of amphetamines by capillary electrophoresis and liquid chromatography : application to drug seizures and cross-validation. Forensic Sci. Int. $1997 ; 86$ : $1-13$.

83. Bjornsdottir I., Hansen S., Fast separation of 16 seizure drug substances using non-aqueous capillary electrophoresis. J. Biochem. Biophys. Methods 1999 ; 38 : 155-61.

84. Wei W., Luo G., Hua G., Yan C., Capillary electrochromatographic separation of basic compounds with bare silica as stationary phase. J. Chromatogr. A $1998 ; 817$ : 65-74.

85. Lurie I., Bailey C., Anex D., Bethea M., McKibben T., Casale J., Profiling of impurities in illicit methamphetamine by high performance liquid chromatography and capillary electrochromatography. J. Chromatogr. A $2000 ; 870(1-2): 53-68$.

86. Harrell C., Dey J., Shamsi S., Foley J., Warner I., Enhanced separation of antidepressant drugs using a polymerised nonionic surfactant as a transient capillary coating. Electrophoresis $1998 ; 19: 712-8$.

87. Deveaux M., Gosset D., Les psychotropes haute couture (designer drugs). Ann. Toxicol. Anal. 2000 ; XII (1):9096.

88. Lurie I., Bethea M., McKibben T., Pellegrini P., Sahat R., Weinberger R., Routine analysis of methamphetamine, amphetamine, end related compounds using capillary electrophoresis. $52^{\text {nd }}$ meeting of American Academy of Forensic Sciences (AAFS), February 21-26, 2000, Reno, NV.

89. Whatley H., Making CE work - Points to consider. LCGC Europe 1999 ; 12 (12) : 762-6.

90. Chapman J., Hobbs J., Putting capillary electrophoresis to work. LC-GC Europe $1999 ; 12$ (5) : 266-79. 